Review

\title{
The Evolving Functions of Autophagy in Ocular Health: A Double-edged Sword
}

\author{
Peiwei Chai ${ }^{*}$, Hongyan $\mathrm{Ni}^{*}$, He Zhang ${ }^{\bowtie \#, ~ X i a n q u n ~ F a n ~}{ }^{\bowtie \#}$ \\ Department of Ophthalmology, Ninth People's Hospital, Shanghai Jiao Tong University School of Medicine, Shanghai, P.R. China. \\ *These authors contributed equally to this paper. \\ \#These authors are co-corresponding authors of this paper. \\ $\square$ Corresponding authors: He Zhang, Ph.D., Ninth People's Hospital, Shanghai JiaoTong University School of Medicine, Shanghai, 200025, P.R. China, E-mail: \\ zhanghe@sjtu.edu.cn; Or Xianqun Fan, M.D, Ph.D., Ninth People's Hospital, Shanghai JiaoTong University School of Medicine, Shanghai, 200025, P.R. China, \\ E-mail: drfanxianqun@126.com.
}

( ) Ivyspring International Publisher. Reproduction is permitted for personal, noncommercial use, provided that the article is in whole, unmodified, and properly cited. See http://ivyspring.com/terms for terms and conditions.

Received: 2016.05.20; Accepted: 2016.08.08; Published: 2016.10.25

\begin{abstract}
Autophagy plays an adaptive role in cell survival, development, differentiation and intracellular homeostasis. Autophagy is recognized as a 'self-cannibalizing' process that is active during stresses such as starvation, chemotherapy, infection, ageing, and oxygen shortage to protect organisms from various irritants and to regenerate materials and energy. However, autophagy can also lead to a form of programmed cell death distinct from apoptosis.

Components of the autophagic pathway are constitutively expressed at a high level in the eye, including in the cornea, lens, retina, and orbit. In addition, the activation of autophagy is directly linked to the development of eye diseases such as age-related macular degeneration (ARMD), cataracts, diabetic retinopathy (DR), glaucoma, photoreceptor degeneration, ocular tumours, ocular infections and thyroid-associated ophthalmopathy (TAO). A high level of autophagy defends against external stress; however, excessive autophagy can result in deterioration, as observed in ocular diseases such as ARMD and DR.

This review summarizes recent developments elucidating the relationship between autophagy and ocular diseases and the potential roles of autophagy in the pathogenesis and treatment of these diseases.
\end{abstract}

Key words: Autophagy, age-related macular degeneration, cataracts, diabetic retinopathy, thyroid-associated ophthalmopathy, ocular tumours.

\section{Introduction to Autophagy}

\section{Underlying autophagic mechanisms}

The word autophagy comes from the Greek words "auto", which means self, and "phagy", which refers to cannibalization. The term "autophagy" refers to any cellular degradative process involving the transport of cytoplasmic cargoes to lysosomes [1]. In a wide range of eukaryotes, autophagy is a cytoprotective process by which a cell "cannibalizes" itself and plays key roles in the basal turnover of cellular components and the response to organelle damage and nutrient deprivation [2].

There are three types of autophagy: macro-autophagy, micro-autophagy and chaperone-mediated autophagy. This review mainly discusses macro-autophagy, which is the most important and canonical mode of autophagy [3]. During this process, cytoplasmic cargoes are sequestered in double-membrane vesicles and delivered to lysosomes. Sequestration vesicles or adaptor proteins (APs) form via a highly regulated process involving more than 20 autophagy-related proteins (Atg). Typical AP formation is highly evolutionarily conserved and proceeds through (1) vesicle nucleation (formation), (2) vesicle elongation (expansion) and (3) vesicle completion (fusion). Table 1 lists molecules involved in the different stages of autophagy and the mechanisms through which they 
function as determined by a number of studies [4-6].

\section{Pathological functions of autophagy}

Autophagy promotes necrosis, tumour progression and the inactivation of apoptosis. Oncogenes such as class I PI3K and Akt activate the mTOR receptor, which inhibits autophagy. In contrast, tumour suppressor genes, such as TSC1, TSC2 and PTEN, trigger autophagy by inhibiting the activity of the mTOR receptor [7-9]. Many neural cells are non-self-renewing and therefore require an appropriate autophagy level to maintain intracellular homeostasis. Autophagy eliminates oxidatively damaged proteins and membranes from the ageing nervous system. Imbalances in autophagy can promote many neurodegenerative disorders, such as Huntington's disease, Parkinson's disease (PD), and Alzheimer's disease (AD) [10-13]. Autophagy is also used as a defence against diverse bacteria and viruses. Numerous medically infectious pathogens are degraded by autophagy, such as group A Streptococcus, herpes simplex virus type I (HSV-1), Listeria monocytogenes, Mycobacterium tuberculosis, Toxoplasma gondii, Shigella flexneri, Francisella tularensis, and Salmonella enterica [14-16]. Autophagy affects Th1/Th2 polarization and can modulate central tolerance, promote the MHC II-mediated presentation of cytosolic antigens, and affect $\mathrm{T}$ and $\mathrm{B}$ cell homeostasis [17].

\section{Autophagic signalling pathways}

The mammalian target of rapamycin, mTOR kinase, is the key inhibitory signal blocking autophagy in the presence of abundant nutrients and growth factors and is the most important autophagic regulator. The activation of mTOR relies on the class I PI3K/Akt signalling pathway, which suppress autophagy as a response to insulin-like growth factor and other growth factors $[7,8]$. Activated mTOR can phosphorylate ULK1/2, thereby suppressing the downstream autophagic cascade [7].

Other autophagic regulatory signalling pathways include (1) eukaryotic initiation factor $2 \mathrm{a}(\mathrm{eIF} 2 \mathrm{\alpha})$, which responds to double-stranded RNA,nutrient deficiency, and endoplasmic reticulum (ER) stress; (2) 5'-AMP-activated protein kinase
(AMPK), which responds to an increased AMP/ATP ratio and a low-energy environment bysuppressing mTORC1 signalling and activating autophagy through TSC1/2 phosphorylation; (3) BH3-only proteins, which contain a Bcl-2 homology-3 (BH3) domain, which interfere with $\mathrm{Bcl}-2 / \mathrm{Bcl}-\mathrm{X}_{\mathrm{L}}-$ mediatedinhibition of the Beclin 1/class III PI3K complex; and (4) P62, which aberrantly accumulates in autophagy-deficient mice and interferes with the association of NRF2 and KEAP1, leading to NRF2 accumulation and stabilization [18-20]. Additionally, the products of more than 20 yeast genes (the ATG genes) act downstream of TOR kinase, and these proteins have complicated functions, some of which remain unknown [3] (see Figure 1).

\section{Autophagy and Retinal Diseases}

Autophagy-related proteins are highly expressed in the retina, including in the ganglion cell layer, the inner nuclear layer, the outer nuclear layer and the retinal pigmented epithelium (RPE) layer. The cells in these layers have a high energy demand and are susceptible to mitochondrial damage [13,21]. Autophagy is responsible for degrading cellular components, and any autophagic defects will disrupt intracellular homeostasis and can cause specific retinal diseases. Two types of retinal disorders related to autophagy are discussed below.

\section{Autophagy and ARMD}

Age-related macular degeneration (ARMD) is triggered by various risk factors, including ageing, obesity, hypercholesterolaemia, hypertension, smoking, arteriosclerosis, and excessive exposure to sunlight. Twenty-five per cent of people over 65 years of age are affected by ARMD, and this condition is a major cause of vision loss in developed countries $[13,21]$. There are two types of ARMD: (1) a dry, atrophic form and (2) a wet exudative form characterized by neovascularization. Patients with the dry form of ARMD generally experience a gradual loss of vision with central visual scotomas that slowly leads to the complete loss of central vision over several years, whereas patients with the wet form of ARMD suffer rapid vision loss [22].

Table 1. Diverse molecular mechanisms functioning at different autophagic stages [4-6].

\begin{tabular}{|c|c|c|}
\hline Stage of autophagy & Molecules involved & Function \\
\hline Nucleation & $\begin{array}{l}\text { BCL-2 interacting myosin/moesin-like coiled-coiled protein } \\
\text { (Beclin 1), the phosphatidylinositol 3-kinase Vps34, and Atg14 }\end{array}$ & $\begin{array}{l}\text { The Beclin1-Vps34-Atg14 complex regulates the production of PtdIns3P } \\
\text { and recruits WIPI1 and Atg2 to form nascent autophagosomes [4]. }\end{array}$ \\
\hline $\begin{array}{l}\text { Elongation and } \\
\text { closure }\end{array}$ & $\begin{array}{l}\text { Atg7-dependent Atg12-Atg5 conjugation system, } \\
\text { microtubule-associated protein } 1 \text { light chain } 3 \text { (LC3), and } \\
\text { phosphatidylethanolamine (PE) }\end{array}$ & $\begin{array}{l}\text { The Atg7-dependent Atg12-Atg } 5 \text { conjugation system is responsible for the } \\
\text { lipidation of LC3 by PE. Lipids are supplied to the expanding } \\
\text { autophagosomal membrane via an Atg9-dependent pathway }[4,5] \text {. }\end{array}$ \\
\hline Degradation & FYVE coiled-coil domain-containing 1 (FYCO1) protein & $\begin{array}{l}\text { FYCO1 functions as a Rab7 effector to mediate the microtubule-dependent } \\
\text { transport of autophagic vacuoles [6]. }\end{array}$ \\
\hline
\end{tabular}




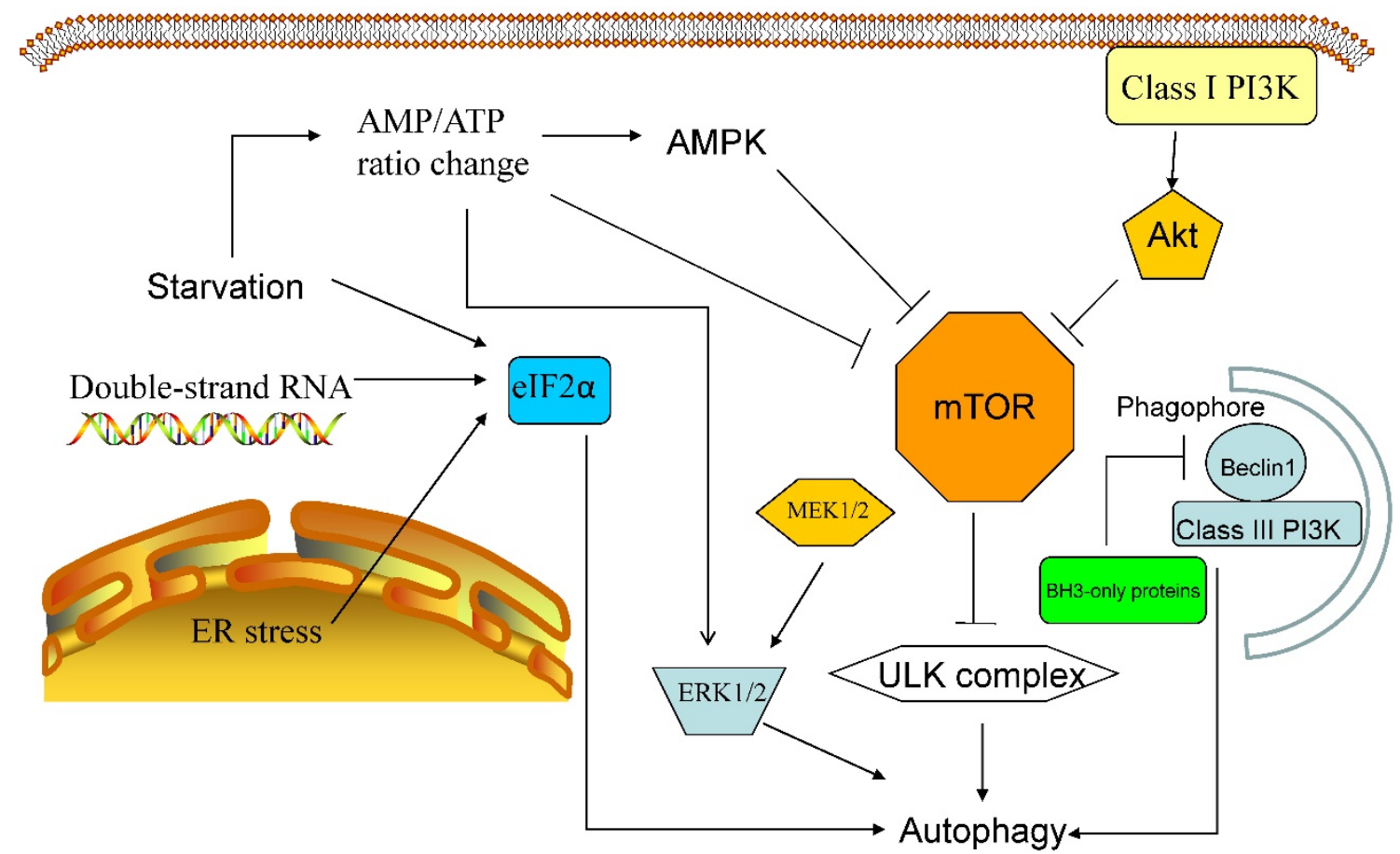

Figure 1. Major autophagic regulatory signalling pathways. The canonical autophagic regulatory pathway is the class I PI3K-Akt-mTOR pathway.The mTOR receptor is a sensor that monitorsintracellularoxygenand nutrient levels andactivatesautophagyin response to hypoxia, ER stressorstarvation; the inhibition of $m$ TOR activates the ULK complex, also resulting in autophagy. Additionally, starvationalters the AMPIATP ratio, leading to the up-regulation of AMPK activity, the activation ofthe ERK $1 / 2$ pathway and the inhibition of the mTOR receptor. Furthermore, ER stress and double-stranded RNA can stimulate elF2 $\alpha$-mediated autophagy, and BH3-only proteins inhibit the Beclin 1/class III PI3K complexwhen theBH3 domain binds to $\mathrm{Bcl}-2 / \mathrm{Bcl}-\mathrm{X}_{\mathrm{L}}$.

The pathology of ARMD is related to inflammation, oxidative stress, and defective lysosomal or proteasomal function, leading to the accumulation of abnormal intra- and extracellular products. The lesions in ARMD are located in the macula, the central and posterior portion of the retina that includes the RPE and photoreceptors. The disease is characterized by the accumulation of drusen, which are abnormal extracellular deposits along the basal layer of the RPE $[13,23,24]$, and drusen deposition is correlated with RPE damage and the degeneration of photoreceptors. Another pathologic feature of ARMD is the accumulation of debris known as lipofuscin in the lysosomes of RPE cells. Lipofuscin, which is a heterogeneous mixture of lipoproteins, sensitizes RPE cells to light exposure and induced oxidative stress, eventually resulting in further protein misfolding $[13,23,24]$.

Autophagy clearly plays a protective role against diseases of the retina and RPE [13]. RPE cells are post-mitotic phagocytes that are non-self-renewing; therefore, the autophagy of these cells' intracellular components is essential for normal cellular function. It was recently found that a high baseline autophagic level is maintained in the retina, in particular in the RPE and photoreceptors, of wild-type mice and that light exposure causes an autophagic response [25]. During the early stages of ARMD, the autophagic level is elevated to compensate for damaged organelles and the results of oxidative stress. In human ARMD samples and mouse ARMD models, the levels of LC3, ATG9 and ATG7 are increased in the RPE and retinal layers [26]. However, in samples from advanced ARMD, the levels of LC3, ATG9 and ATG7 are decreased, and we assume that the lack of autophagic activity causes the late-stage disease [13].

N-retinylidene-N-retinylethanolamine (A2E), which is deposited in the RPE cells of older patients, is a major toxic lipofuscin component.Zhang et al. found that A2E triggers autophagy in RPE cells during the early stages of ARMD. An increased autophagic level in RPE cells protects against the harmful effects of A2E by reducing the secretion of inflammatory cytokines and VEGFA.A decrease to autophagic levels makes RPE cells susceptible to apoptosis induced by various stresses, including nutritional depletion and mitochondrial photooxidative damage [27].

Lysosomes are the most important digestive organelles in eukaryotic cells. The degradative phase of autophagy occurs in lysosomes, to which cytoplasmic cargoes are delivered [28]. Defective lysosomal degradation is the major cause of lipofuscinogenesis. Animal models deficient in bA3/A1-crystallin, which is encoded by the Cryba1 gene, exhibit insufficient lysosomal clearance in RPE cells, the same pathological sign seen in AMD patients [29].bA3/A1-crystallin regulates lysosome-mediated degradation in the RPE by modulating the activity of 
V-ATPase, the proton pump that acidifies the lysosomal lumen, through the AKT/mTORC1 signalling pathway [30,31].Deficient lysosomal function will disrupt the autophagic degradation of toxic components and misfolded proteins.

\section{Autophagy and DR}

Diabetic retinopathy (DR) is a serious complication of diabetes mellitus. DR is currently one of the major causes of blindness, and its prevalence is dramatically increasing worldwide. DR affects up to $80 \%$ of patients with a 20 year or longer history of diabetes. The ocular complications of DR include damaged visual acuity and eventual blindness. Diabetic retinopathy is a retinal microangiopathy [32], and damage to the pericytes on the retinal capillaries is evident during the early stages of DR. Retinal capillary occlusion resulting in elevated vascular permeability and ischaemia of the retina causes breaks in the blood-retina barrier and angiographic leakage [33].

The role of autophagy in DR is quite complicated. Autophagy promotes pericyte survival in early DR, whereas excessive autophagy causes excess stress and leads to necrosis [34,35]. High blood glucose levels also trigger autophagy to prevent glucose-induced damage in the RPE. In this scenario, autophagy is mainly regulated through ROS-mediated ER stress instead of through the mTOR signalling pathway [36]. Moreover, a damaged blood-retina barrier releases cytoplasmic lipoproteins, and extra-vascular modified LDL promotes RPE damage through ER stress, oxidative stress, apoptosis and autophagy. In this case, autophagy is detrimental to DR treatment [34], suggesting that autophagy might be a potential target to treat DR.

\section{Autophagy and chloroquine retinopathy}

Chloroquine (CQ) and hydroxychloroquine (HCQ) are widely used for the treatment of rheumatic diseases such as rheumatic arthritis (RA), Sjogren's syndrome and malaria. The major complication of treatment with HCQ and/or CQ is retinopathy with subsequent permanent visual damage [37]. Patients who experience this complication develop different types of visual damage, including pericentral, paracentral, central, and/or peripheral field loss. The optimal $\mathrm{pH}$ of healthy lysosomes is acidic $(\mathrm{pH}<5)$; in fact, an acidic lysosomal $\mathrm{pH}$ is required for efficient autophagic degradation in RPE cells [38].

Treatment with HCQ or CQ will cause the alkalinization of lysosomes, resulting in disturbance in the enzymatic clearance of autophagosomes [39]. Although such treatment produces only a small increase in lysosomal $\mathrm{pH}$, autophagic degradation will be strongly inhibited. Similarly to ARMD, protease dysfunction gives rise to the accumulation of lipofuscin and A2E in RPE cells [27]. In addition, treatment with CQ and/or HCQ also increases the mRNA expression levels of transcription factor EB (TFEB) and the proton pump $\mathrm{v}-\left(\mathrm{H}^{+}\right)$ATPase [40]. Similar features are observed in AD patients with fibroblasts with mutant presenilin 1 (PS1), suggesting a potential link between ARMD and AD [41].

\section{Autophagy and Orbital Disease: Thyroid- associated Ophthalmopathy (TAO)}

Grave's disease (GD) is an autoimmune-inflammatory disorder characterized by the release of autoantibodies directed at the thyrotropin receptor of thyrocytes, leading to the activation of thyrocyte function and the overproduction of thyroid hormones [42]. Approximately $50 \%$ of GD patients develop thyroid-associated ophthalmopathy (TAO), which is the most prevalent orbital disease. GD patients generally develop ocular symptoms such as eye irritation, redness, excessive tearing, photophobia, double vision, and a sensation of pressure behind the eyes. Patients with severe GD suffer vision loss due to compressive optic neuropathy or cornea ulceration [43]. However, few reports have associated autophagy with TAO.

Yoon et al. found that GD tissues exhibit a high level of autophagic activity compared to non-GD counterparts. Inflammatory cytokines, such as interleukin-1b, can also trigger autophagy. The authors also showed that APs accumulated with lipid droplets in GD tissues. Bafilomycin A1 treatment and knockdown of Atg5 expression via shRNA suppressed lipid-droplet accumulation and inhibited the expression of adipogenic markers [44]. Additionally, statin treatment decreases the risk of orbitopathy in patients with GD by regulating apoptosis and autophagic activities [45].

Although few reports have suggested a relationship between TAO and autophagy, numerous articles have revealed that autophagy can lead to fibrosis in the liver; a similar pathological feature is observed in TAO. Autophagy markers are markedly upregulated in the livers of cirrhotic patients [46]. However, autophagy is antifibrotic and can be protective against fibrosis. For example, in mice, a mutation in Atg5, an autophagy gene, interfered with chronic liver fibrosis [47]. In addition to fibrosis, adipogenesis is a pathological feature of TAO. Autophagy can also promote the differentiation of fat tissue. Atg7 knock-out mice present with little adipose tissue and greatly increased numbers of mitochondria $[48,49]$. Further research examining this subject is 
urgently needed.

\section{Autophagy and Glaucoma}

Glaucoma, a neurodegenerative disorder that is characterized by damage to the retinal ganglion cells (RGCs) and the axons of the optic nerve, is the second leading cause of permanent blindness [50]. This disorder is divided into two main categories, open-angle glaucoma and closed-angle glaucoma, based on the iris-cornea angle. Open-angle glaucoma is generally painless, tends to develop slowly over time and seldom has symptoms until the disease has significantly progressed [51]. Closed-angle glaucoma can be asymptomatic but present suddenly with abrupt eye pain, blurred vision, redness, vomiting, and nausea. Glaucoma used to be regarded as an optic neuropathy caused by increased intraocular pressure (IOP); however, the elevated IOP results from the occlusion of the trabecular meshwork (TM) that inhibits the outflow of aqueous humour. The decreased aqueous-humour outflow leads to optic nerve and RGC damage [50,52].

As in other neurodegenerative diseases such as $\mathrm{AD}$ and PD, deficient autophagy causes the accumulation of damaged cytoplasmic proteins and excessive oxidative stress. Optic-nerve axotomy generates an acute model of glaucoma that can be used to investigate its effect on RGCs. Autophagy promotes the survival of RGCs after an optic-nerve axotomy [53]. Like neurons, TM cells are post-mitotic, non-self-renewing cells that require a high level of autophagy to maintain intracellular homeostasis. Furthermore, TM cells are exposed to a high level of ROS, leading to the accumulation of non-degradable material in lysosomes, which subsequently decreases lysosomal activity. When TM cells are exposed to external stresses, such as high pressure or biaxial static stretching, autophagy is activated to eliminate damaged proteins and organelles. Decreased autophagic flux is a symbol of progressive TM dysfunction [54,55], and regulating autophagic activity is a new strategy to treat retinal diseases that can cause irreversible neural degeneration [56].

However, autophagy sometimes plays a deleterious role in glaucoma pathogenesis [57]. Elevated IOP can activate autophagy, up-regulating the levels of autophagic markers such as LC3-II/LC3-I and beclin- 1 and inducing cell death. Inhibiting autophagy via 3-MA treatment can inhibit apoptosis [58]. It is assumed that during the early stages of glaucoma, the stress of an elevated IOP activates dendritic autophagy. During the later stages of the disease, elevated IOP leads to the autophagy of cytoplasmic components, resulting in apoptosis.

\section{Autophagy and Cataracts}

A cataract is a clouding of the lens that leads to decreased visual acuity and can develop slowly in one eye or in both eyes. The symptoms include colours appearing faded, halos around light sources, blurry vision, and difficulty with bright lights. Cataracts are the cause of up to half of blindness cases and one-third of visual impairment cases worldwide. The anterior pole of the lens is covered by a simple cuboidal epithelium, the cells of which continuously differentiate to form fibre cells [59]. To maintain the transparency of the lens, these fibre cells degrade their organelles to create an organelle-free zone (OFZ) [60].

Autophagy is a key regulator of OFZ formation and lens fibre cell maturation. Serum starvation can activate autophagy and mitophagy in the embryonic and adult lens, suggesting that lens cells respond to environmental stress through autophagy and mitophagy [60].

Congenital cataracts are directly linked to mutations in the FYCO1 gene. FYCO1 is a PI(3)P-, Rab7- and LC3-binding protein that mediates the transport of APs, an essential process for autolysosome formation. Mutation of the FYCO1 gene can lead to an autosomal-recessive form of congenital cataracts [60]. Additionally, Vps34is involved in Atg5-independent autophagy, and a mutation in Vps34can also lead to the formation of congenital cataracts [61]. The R120G mutation in aB-crystallin increases the levels of LC3-II and p62 and the size of the APs in lens fibre and epithelial cells in a hereditary cataract model [62]. In conclusion, the disruption of autophagy in lens cells results in a loss of stress resistance and inhibits differentiation, finally resulting in cataract formation.

\section{Autophagy and Ocular Infections}

The direct exposure of the eye to the environment coupled with the unique structure of the human eye makes it vulnerable to many infectious diseases. After the anatomical barriers of the eye are breached, humans do not mount a sufficient immune response to prevent the infection from spreading because of a specific ocular immune-tolerance area, and such infections can eventually result in blindness. The human cornea is the ideal target for herpes simplex virus 1 (HSV-1) and T. gondii, which enter endothelial cells via endocytosis [63].

Autophagy is crucial to degrade infectious microorganisms. T. gondii is eliminated by macrophages via CD40, which induces the autophagic killing process. Ocular toxoplasmosis generally occurs in immunocompromised and immune-incompetent patients, most often resulting in posterior uveitis. 
Patients with defective CD40 generally develop ocular toxoplasmosis. CD40 recruits APs to the area surrounding parasitophorous vacuoles, which induces macrophages to eliminate $T$. gondii cells [64-66]. HSV-1, a major cause of viral keratitis, is a double-stranded DNA virus that inhibits the corneal response to infection by inhibiting autophagic activity [67]. PKR- and eIF2a-dependent autophagy degrades the HSV-1 virus, although HSV-1 produces ICP34.5, a Beclin 1 antagonist that represses PKR-mediated eIF2a phosphorylation and prevents autophagic degradation [68]. In summary, it is believed that autophagy plays a protective role against various infectious diseases by rapidly inducing the innate immune response.

\section{Autophagy and Ocular Cancer}

There are two categories of ocular cancer, primary cancer (which begins in the eye) and metastatic cancer (which has spread from other organs). The most common primary ocular cancer in adults is uveal melanoma, which can arise in the choroid, ciliary body and iris. The most common ocular cancer in children is retinoblastoma, which affects 325 children in the USA per year [69-71]. The relationship between autophagy and cancer is complicated. First, autophagy can help cancer cells cope with excessive environmental stresses, such as inflammation, hypoxia, and high ROS levels. During chemotherapy or radiotherapy treatment, an elevated level of autophagy promotes cancer cell survival. However, several types of tumours can be induced to undergo apoptosis or necrosis when the level of autophagy is increased, indicating that autophagy can also be detrimental to cancer cell proliferation.

The autophagic activity in 99 uveal melanoma patients was investigated using the autophagic markers LC3A and Beclin 1. In uveal melanomas, autophagy is commonly up-regulated, which might be related to hypoxia and intense pigmentation. The overexpression of Beclin 1 in uveal melanoma patients is associated with early metastasis and a poor diagnosis [72]. Moreover, AKT and MEK inhibition can also induce AMPK-dependent autophagic cell death, making this pathway a promising target for the treatment of uveal melanoma [73]. MicroRNA 34a (MIR34A), functions as a tumour-suppressor gene in retinoblastoma cells, down-regulates the level of high-mobility group box 1(HMGB1) protein and enhances chemotherapy-induced apoptosis. MIR34A inhibits HMGB1, resulting in decreased autophagic activity during starvation and chemotherapy. Thus, up-regulating MIR34A levels, inhibiting HMGB1 activity or repressing autophagic activity might restore the chemosensitivity of retinoblastoma cells and induce cell death [74].

\section{Conclusion}

Despite being viewed as a degradative process, autophagy also regenerates raw material and recycles energy to maintain intracellular homeostasis. Autophagy clearly functions as a dual-edged weapon in the pathogenesis of ocular diseases. The level of autophagy is elevated when cells are subjected to growth-factor deprivation or to glucose or oxygen shortage $[2,3,75]$, and cells lose the majority of their mass through autophagy of their components under these circumstances. When such cells are placed in optimal conditions, they become more sensitive to nutrients and once again exhibit the ability to uptake and metabolize glucose. In this case, autophagy is inhibited in response to growth factors or nutrients. During this period in optimal conditions, cells recover their proliferative potential and return to their original size [76]. On the other hand, autophagy promotes cell deaths and causes deterioration in certain diseases. Knocking down the Beclin 1 gene or the Atg gene blocks non-apoptotic cell death induced by caspase- 8 inhibition in L929 fibrosarcoma cells and U937 monocytoid cells [77]. Tat-Beclin 1, an autophagy-inducing peptide, causes cell death with morphological features characteristic of autophagy [78].

In most cases, autophagy is responsible for metabolic regulation and intracellular recycling. Changes to autophagic activity are correlated with the development of various ocular-tissue disorders (see Figure 2). We discussed the protective role that autophagy plays in the pathogenesis of ARMD, DR, glaucoma, cataracts, ocular infections and ocular cancers. However, excessive autophagy also occurs in $\mathrm{DR}$, glaucoma, TAO, and ocular cancers, and excessive autophagic activity ultimately results in apoptosis and necrosis. Unlike self-renewing cells, neurons and lens fibre cells require a high level of autophagic activity to eliminate toxic metabolic waste products and misfolded proteins. When autophagy is inhibited, environmental stress leads to the degeneration of these post-mitotic cells. In the case of ocular tumours, autophagy can promote tumour cell survival during chemotherapy and radiotherapy treatment, and a high level of autophagy is associated with a poor prognosis and early metastasis. Thus, ocular tumours might be effectively treated by combining chemotherapeutic drugs and autophagy inhibitors (see Table 2). In brief, regulating autophagic activity in various ocular diseases is a promising treatment strategy. 


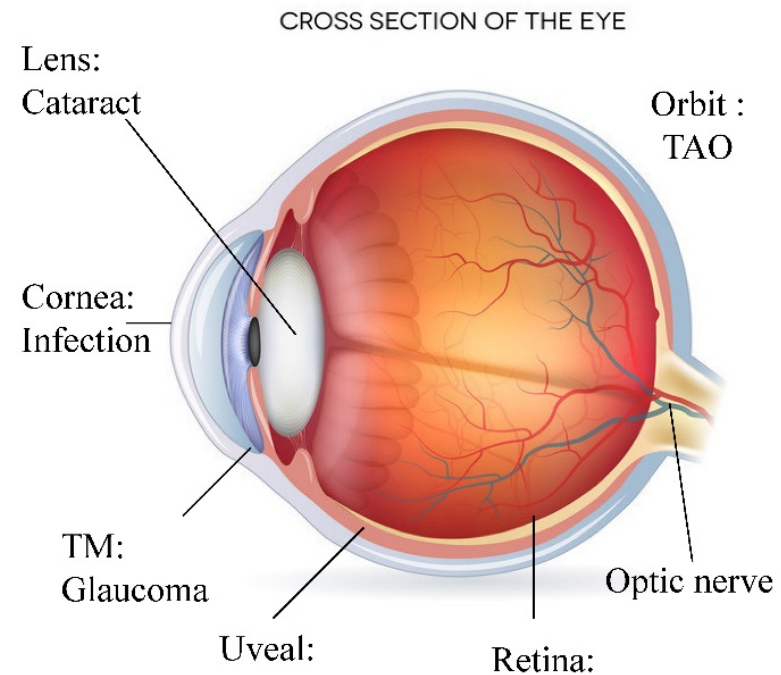

Melanoma

ARMD, DR,
VIEW OF FUNDUS

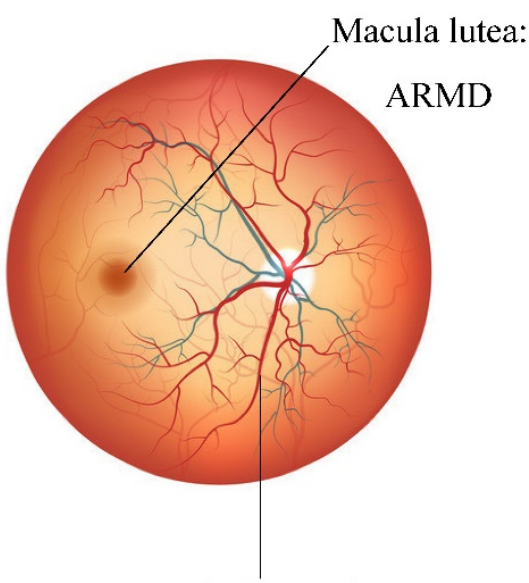

Retinal vascular:

RD

retinoblastoma and

glaucoma

Figure 2. Ocular diseases associated with autophagy. Various ocular diseases involve an imbalance in autophagic activity. The major disorders affected by autophagy level are neurodegenerative and infectious diseases. Ocular tumours, such as uveal melanoma and retinoblastoma, are also associated with misregulated autophagy, which results in the storage of energy and materials that can be used for uncontrolled proliferation. Imbalanced autophagy causes keratitis, glaucoma, congenital cataracts, DR, ARMD, TAO and ocular tumours.

Table 2. Autophagic changes that occur in different ocular regions, disorders and autophagy-related treatments.

\begin{tabular}{|c|c|c|c|}
\hline $\begin{array}{l}\text { Ocular } \\
\text { region }\end{array}$ & $\begin{array}{l}\text { Autophagy-related } \\
\text { diseases }\end{array}$ & Description & $\begin{array}{l}\text { Autophagy-related } \\
\text { treatment }\end{array}$ \\
\hline Cornea & Corneal infections & $\begin{array}{l}\text { HSV-1 infection inhibits the normal autophagic response. Autophagic dysregulation } \\
\text { results from a CD } 40 \text { pathway defect, promoting T. gondii infections [64-68]. }\end{array}$ & $\begin{array}{l}\text { Induce autophagy to maintain } \\
\text { intracellular homeostasis }\end{array}$ \\
\hline Lens & Cataracts & $\begin{array}{l}\text { Autophagy promotes autophagosome transport. Deficient degradation leads to } \\
\text { congenital cataracts. Increased levels of LC3-II and autophagosomes in a hereditary } \\
\text { cataract model }[60,62] \text {. }\end{array}$ & \\
\hline TM & Glaucoma & High-pressure stress and biaxial static stretching triggers autophagy [55]. & \\
\hline Uvea & Uveal melanoma & $\begin{array}{l}\text { High-level autophagic activity is an indicator of poor prognosis and early metastasis } \\
\text { [72]. }\end{array}$ & Inhibit autophagy \\
\hline \multirow[t]{4}{*}{ Retina } & ARMD & $\begin{array}{l}\text { Insufficient lysosomal degradation and decreased autophagic activity results in } \\
\text { ARMD. Additionally, autophagy decreases the level of inflammatory compounds } \\
\text { [25]. }\end{array}$ & $\begin{array}{l}\text { 1. Induce autophagy via rapamycin, an } \\
\text { AMPK activator, RAP or MG132. } \\
\text { 2. Inhibit autophagy via chloroquine or } \\
\text { hydroxychloroquine. } \\
\text { Each strategy has been shown to cure } \\
\text { ARMD. }\end{array}$ \\
\hline & DR & $\begin{array}{l}\text { Autophagy promotes pericyte survival to a certain extent. Altered expression of p62 } \\
\text { and LC3 is observed in DR patients [36]. }\end{array}$ & Induce autophagy \\
\hline & Glaucoma & Glaucoma triggers autophagy in RGCs [53]. & \\
\hline & Retinoblastoma & $\begin{array}{l}\text { MIR34A inhibits HMGB1, decreasing autophagic activity upon starvation or } \\
\text { chemotherapeutic treatment [74]. }\end{array}$ & $\begin{array}{l}\text { Inhibit autophagy via MIR34A to maintain } \\
\text { sensitivity to chemotherapeutics }\end{array}$ \\
\hline Orbit & TAO & $\begin{array}{l}\text { High-level expression of autophagic markers in TAO patients. Autophagic inhibitors } \\
\text { decrease the adipogenic capacity of fibroblasts in TAO. Statins reduce the risk of } \\
\text { orbitopathy in patients with Graves' disease by modulating apoptosis and autophagic } \\
\text { activity }[44,45] \text {. }\end{array}$ & $\begin{array}{l}\text { Inhibit autophagy via } \\
\text { bafilomycin A1 }\end{array}$ \\
\hline
\end{tabular}

\section{Acknowledgements}

This work was supported by the Scientific Research Program of the National Health and Family Planning Commission of China (201402014), the National Natural Science Foundation of China (31470757), the Program for Professors of Special Appointment (Eastern Scholar) at the Shanghai Institutions of Higher Learning (1410000159), the
SMC-ChenXing Young Scholar Program (2014, Class B), the Shanghai Municipal Education CommissionGaofeng Clinical Medicine Grant (2016-13) and the Science and Technology Commission of Shanghai (14JC1404100, 14JC1404200, and 14430723100). The funders of this research had no role in study design, data collection and analysis, the decision to publish, or the preparation of the manuscript. 


\section{Conflicts of interest}

The authors declare no conflicts of interest.

\section{References}

1. Shintani T, Klionsky DJ. Autophagy in health and disease: a double-edged sword. Science. 2004; 306: 990-5.

2. Mizushima N, Levine B, Cuervo AM, et al. Autophagy fights disease through cellular self-digestion. Nature. 2008; 451: 1069-75.

3. Levine B, Kroemer G. Autophagy in the pathogenesis of disease. Cell. 2008; 132: $27-42$.

4. Walker S, Chandra P, Manifava M, et al. Making autophagosomes: localized synthesis of phosphatidylinositol 3-phosphate holds the clue. Autophagy. 2008; 4: 1093-6.

5. Boya P, Reggiori F, Codogno P. Emerging regulation and functions of autophagy. Nat Cell Biol. 2013; 15: 713-20.

6. Pankiv S, Alemu EA, Brech A, et al. FYCO1 is a Rab7 effector that binds to LC3 and PI3P to mediate microtubule plus end-directed vesicle transport. J Cell Biol. 2010; 188: 253-69.

7. Kim J, Kundu M, Viollet B, et al. AMPK and mTOR regulate autophagy through direct phosphorylation of Ulk1. Nat Cell Biol. 2011; 13: 132-41.

8. Zoncu R, Efeyan A, Sabatini DM. mTOR: from growth signal integration to cancer, diabetes and ageing. Nat Rev Mol Cell Biol. 2010; 12: 21-35.

9. Mihaylova MM, Shaw RJ. The AMPK signalling pathway coordinates cell growth, autophagy and metabolism. Nat Cell Biol. 2011; 13: 1016-23.

10. Nixon RA. The role of autophagy in neurodegenerative disease. Nat Med. 2013; 19: 983-97.

11. Hornung JP, Koppel H, Clarke PGH. Endocytosis and autophagy in dying neurons: an ultrastructural study in chick embryos. J Comp Neurol. 1989; 283: 425-37.

12. Kawashima M, Ozawa Y, Shinmura K, et al. Calorie restriction (CR) and CR mimetics for the prevention and treatment of age-related eye disorders. Exp Gerontol. 2013; 48: 1096-100.

13. Mitter SK, Song C, Qi X, et al. Dysregulated autophagy in the RPE is associated with increased susceptibility to oxidative stress and AMD. Autophagy. 2014; 10: 1989-2005.

14. Portillo J-AC, Okenka G, Reed E, et al. The CD40-autophagy pathway is needed for host protection despite IFN- $\Gamma$-dependent immunity and CD40 induces autophagy via control of P21 levels. PLoS One. 2010; 5: e14472.

15. Levine B, Deretic V. Unveiling the roles of autophagy in innate and adaptive immunity. Nat Rev Immunol. 2007; 7: 767-77

16. Leib DA, Alexander DE, Cox D, et al. Interaction of ICP34.5 with beclin 1 modulates herpes simplex virus type 1 pathogenesis through control of CD4+ T-cell responses. J Virol. 2009; 83: 12164-71.

17. Harris J, Master SS, de Haro SA, et al. Th1-Th2 polarisation and autophagy in the control of intracellular mycobacteria by macrophages. Vet Immunol Immunopathol. 2009; 128: 37-43.

18. Criollo A, Maiuri MC, Tasdemir E, et al. Regulation of autophagy by the inositol trisphosphate receptor. Cell Death Differ. 2007; 14: 1029-39.

19. Maiuri MC, Zalckvar E, Kimchi A, et al. Self-eating and self-killing: crosstalk between autophagy and apoptosis. Nat Rev Mol Cell Biol. 2007; 8: 741-52.

20. Wang $\mathrm{Y}$, Huang $\mathrm{C}$, Zhang $\mathrm{H}$, et al. Autophagy in glaucoma: crosstalk with apoptosis and its implications. Brain Res Bull. 2015; 117: 1-9.

21. Mo J, Zhang M, Marshall B, et al. Interplay of autophagy and apoptosis during murine cytomegalovirus infection of RPE cells. Mol Vis. 2014; 20: 1161-73.

22. Weir E. Age-related macular degeneration: armed against ARMD. CMAJ. 2004; 170: 463-4

23. de Jong PTVM. Drusen, AMD, and history. Graefes Arch Clin Exp Ophthalmol. 2015; 253: 2061-2.

24. Fanjul-Moles ML, López-Riquelme GO. Relationship between oxidative stress, circadian rhythms, and AMD. Oxid Med Cell Longev. 2016; 2016: 1-18.

25. Chen $\mathrm{Y}$, Sawada $\mathrm{O}, \mathrm{Kohno} \mathrm{H}$, et al. Autophagy protects the retina from light-induced degeneration. J Biol Chem. 2013; 288: 7506-18.

26. Wang AL, Lukas TJ, Yuan M, et al. Autophagy and exosomes in the aged retinal pigment epithelium: possible relevance to drusen formation and age-related macular degeneration. PLoS One. 2009; 4: e4160.

27. Zhang J, Bai Y, Huang L, et al. Protective effect of autophagy on human retinal pigment epithelial cells against lipofuscin fluorophore A2E: implications for age-related macular degeneration. Cell Death Dis. 2015; 6: e1972.

28. Ward C, Martinez-Lopez N, Otten EG, et al. Autophagy, lipophagy and lysosomal lipid storage disorders. Biochim Biophys Acta. 2016; 1861: 269-84.

29. Sinha D, Klise A, Sergeev $Y$, et al. $\beta A 3 / A 1$-crystallin in astroglial cells regulates retinal vascular remodeling during development. Mol Cell Neurosci. 2008; 37: 85-95.

30. Valapala M, Edwards M, Hose S, et al. Increased Lipocalin-2 in the retinal pigment epithelium of Cryba1 cKO mice is associated with a chronic inflammatory response. Aging Cell. 2014; 13: 1091-4.

31. Valapala M, Wilson C, Hose S, et al. Lysosomal-mediated waste clearance in retinal pigment epithelial cells is regulated by CRYBA1/ $\beta A 3 / A 1-c r y s t a l l i n$ via V-ATPase-MTORC1 signaling. Autophagy. 2014; 10: 480-96.

32. Ebneter A, Zinkernagel MS. Novelties in diabetic retinopathy. Endocr Dev. 2016; 31: 84-96.
33. Cai X, McGinnis JF. Diabetic retinopathy: animal models, therapies, and perspectives. J Diabetes Res. 2016; 2016: 1-9.

34. Fu D, Wu M, Zhang J, et al. Mechanisms of modified LDL-induced pericyte loss and retinal injury in diabetic retinopathy. Diabetologia. 2012; 55: 3128-40.

35. Du M, Wu M, Fu D, et al. Effects of modified LDL and HDL on retinal pigment epithelial cells: a role in diabetic retinopathy? Diabetologia. 2013; 56: 2318-28.

36. Yao J, Tao Z-F, Li C-P, et al. Regulation of autophagy by high glucose in human retinal pigment epithelium. Cell Physiol Biochem. 2014; 33: 107-16.

37. Ganne P, Srinivasan R. Chloroquine retinopathy. JAMA Ophthalmol. 2015; 133: 603-4.

38. Guha S, Coffey EE, Lu W, et al. Approaches for detecting lysosomal alkalinization and impaired degradation in fresh and cultured RPE cells: evidence for a role in retinal degenerations. Exp Eye Res. 2014; 126: 68-76.

39. Zhan J, He J, Zhou Y, et al. Crosstalk between the autophagy-lysosome pathway and the ubiquitin-proteasome pathway in retinal pigment epithelial cells. Curr Mol Med. 2016; 16: 487-95.

40. Coffey EE, Beckel JM, Laties AM, et al. Lysosomal alkalization and dysfunction in human fibroblasts with the alzheimer's disease-linked presenilin 1 A246E mutation can be reversed with cAMP. Neuroscience. 2014; 263: 111-24.

41. Lee J-H, Yu WH, Kumar A, et al. Lysosomal proteolysis and autophagy require presenilin 1 and are disrupted by alzheimer-related PS1 mutations. Cell. 2010; 141: 1146-58.

42. Bahn RS. Graves' ophthalmopathy. N Engl J Med. 2010; 362: 726-38.

43. Eckstein A, Berchner-Pfannschmidt U, Führer D, et al. Update endokrine orbitopathie. Ophthalmologe. 2013; 110: 1079-96

44. Yoon JS, Lee HJ, Chae MK, et al. Autophagy is involved in the initiation and progression of graves' orbitopathy. Thyroid. 2015; 25: 445-54.

45. Bifulco M, Ciaglia E. Statin reduces orbitopathy risk in patients with graves' disease by modulating apoptosis and autophagy activities. Endocrine. 2015. doi: 10.1007/s12020-015-0762-z

46. Mallat A, Lodder J, Teixeira-Clerc F, et al. Autophagy: a multifaceted partner in liver fibrosis. Biomed Res Int. 2014; 2014: 1-7.

47. Lodder I, Denaës T, Chobert M-N, et al. Macrophage autophagy protects against liver fibrosis in mice. Autophagy. 2015; 11: 1280-92.

48. Guo L, Huang J-X, Liu Y, et al. Transactivation of Atg4b by C/EBP $\beta$ promotes autophagy to facilitate adipogenesis. Mol Cell Biol. 2013; 33: 3180-90.

49. Zhang $Y$, Goldman $S$, Baerga $R$, et al. Adipose-specific deletion of autophagy-related gene 7 (atg7) in mice reveals a role in adipogenesis. Proc Natl Acad Sci. 2009; 106: 19860-5.

50. Kim KE, Park K-H. Update on the prevalence, etiology, diagnosis, and monitoring of normal-tension glaucoma. Asia Pac J Ophthalmol. 2016; 5: 23-31.

51. Chang RT, Singh K. Glaucoma suspect: diagnosis and management. Asia Pac J Ophthalmol. 2016; 5: 32-7.

52. Lam D, Lee J, Jonas J, et al. Glaucoma: today and tomorrow. Asia Pac J Ophthalmol. 2016; 5: 2-4

53. Rodríguez-Muela N, Germain F, Mariño G, et al. Autophagy promotes survival of retinal ganglion cells after optic nerve axotomy in mice. Cell Death Differ. 2011; 19: 162-9.

54. Porter K, Nallathambi J, Lin Y, et al. Lysosomal basification and decreased autophagic flux in oxidatively stressed trabecular meshwork cells. Autophagy. 2013; 9: 581-94.

55. Porter KM, Jeyabalan N, Liton PB. MTOR-independent induction of autophagy in trabecular meshwork cells subjected to biaxial stretch. Biochim Biophys Acta. 2014; 1843: 1054-62.

56. Wang $\mathrm{Y}, \mathrm{Xu} \mathrm{K}$, Zhang $\mathrm{H}$, et al. Retinal ganglion cell death is triggered by paraptosis via reactive oxygen species production: a brief literature review presenting a novel hypothesis in glaucoma pathology. Mol Med Rep. 2014; 10: $1179-83$

57. Knoferle J, Koch JC, Ostendorf $\mathrm{T}$, et al. Mechanisms of acute axonal degeneration in the optic nerve in vivo. Proc Natl Acad Sci. 2010; 107: 6064-9.

58. Park HYL, Kim JH, Park CK. Activation of autophagy induces retinal ganglion cell death in a chronic hypertensive glaucoma model. Cell Death Dis. 2012; 3: e290.

59. El Fkih L, Hmaied W, El Hif S, et al. Congenital cataract etiology. Tunis Med. 2007; 85: 1025-9.

60. Chen J, Ma Z, Jiao X, et al. Mutations in FYCO1 cause autosomal-recessive congenital cataracts. Am J Med Genet. 2011; 88: 827-38.

61. Morishita H, Eguchi S, Kimura H, et al. Deletion of autophagy-related 5 (Atg5) and Pik3c3 genes in the lens causes cataract independent of programmed organelle degradation. J Biol Chem. 2013; 288: 11436-47.

62. Wignes JA, Goldman JW, Weihl CC, et al. p62 expression and autophagy in aB-crystallin R120G mutant knock-in mouse model of hereditary cataract. Exp Eye Res. 2013; 115: 263-73.

63. McCannel CA, Holland GN, Helm CJ, et al. Causes of uveitis in the general practice of ophthalmology. Am J Ophthalmol. 1996; 121: 35-46.

64. Subauste CS, Andrade RM, Wessendarp M. CD40-TRAF6 and autophagy-dependant anti-microbial activity in macrophages. Autophagy. 2007; 3: 245-8.

65. Subauste CS, Wessendarp M. CD40 restrains in vivo growth of Toxoplasma gondii independently of gamma interferon. Infect Immun. 2006; 74: 1573-9.

66. Subauste CS, Wessendarp M, Sorensen RU, et al. CD40-CD40 ligand interaction is central to cell-mediated immunity against Toxoplasma gondii: patients with hyper IgM syndrome have a defective type 1 immune response 
that can be restored by soluble CD40 ligand trimer. J Immunol. 1999; 162: 6690-700.

67. Tallóczy Z, Virgin IVH, Levine B. PKR-dependent xenophagic degradation of herpes simplex virus type 1. Autophagy. 2006; 2: 24-9.

68. Orvedahl A, Alexander D, Tallóczy Z, et al. HSV-1 ICP34.5 confers neurovirulence by targeting the beclin 1 autophagy protein. Cell Host Microbe. 2007; 1: 23-35

69. Little MP. A review of non-cancer effects, especially circulatory and ocular diseases. Radiat Environ Biophys. 2013; 52: 435-49.

70. Fan JY, Xing Y, Wen XY, et al. Long non-coding RNA ROR decoys genespecific histone methylation to promote tumorigenesis. Genome Biology. 2015; 16:139.

71. Xu S, Wang H, Pan $\mathrm{H}$, et al. ANRIL IncRNA triggers efficient therapeutic efficacy by reprogramming the aberrant INK4-hub in melanoma. Cancer Lett. 2016; 381(1):41-8

72. Giatromanolaki AN, Charitoudis G, Bechrakis NE, et al. Autophagy patterns and prognosis in uveal melanomas. Mod Pathol. 2011; 24: 1036-45.

73. Ambrosini G, Musi E, Ho AL, et al. Inhibition of mutant GNAQ signaling in uveal melanoma induces AMPK-dependent autophagic cell death. Mol Cancer Ther. 2013; 12: 768-76.

74. Liu K, Huang J, Xie M, et al. MIR34A regulates autophagy and apoptosis by targeting HMGB1 in the retinoblastoma cell. Autophagy. 2014; 10: 442-52.

75. Kundu M, Thompson CB. Autophagy: basic principles and relevance to disease. Annu Rev Pathol Mech Dis. 2008; 3: 427-55.

76. Lum JJ, Bauer DE, Kong M, et al. Growth factor regulation of autophagy and cell survival in the absence of apoptosis. Cell. 2005; 120: 237-48.

77. Yu L, Alva A, Su H, et al. Regulation of an ATG7-beclin 1 program of autophagic cell death by caspase-8. Science. 2004; 304: 1500-2.

78. Shoji-Kawata S, Sumpter R, Leveno M, et al. Identification of a candidate therapeutic autophagy-inducing peptide. Nature. 2013; 494: 201-6. 